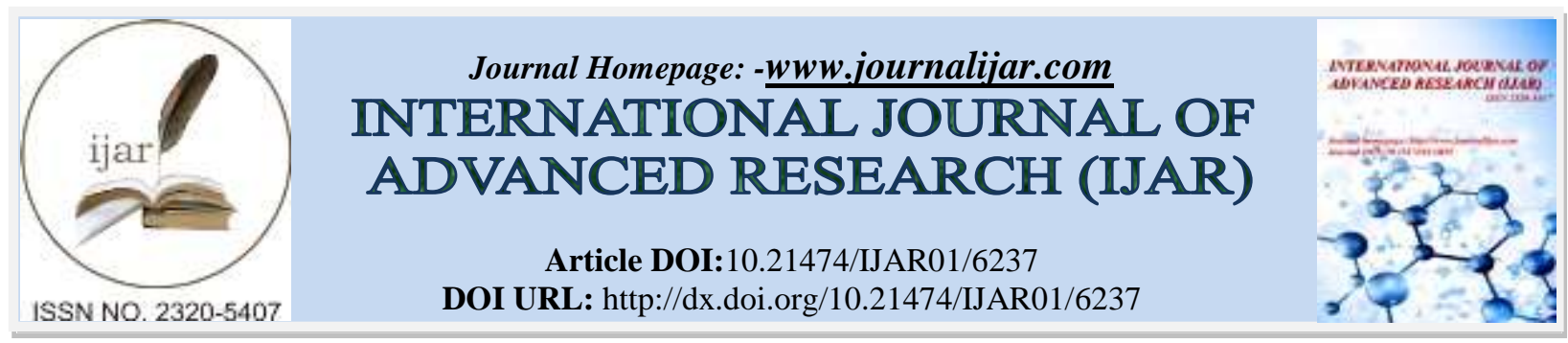

RESEARCH ARTICLE

\title{
EVALUATION OF TOXIC EFFECTS OF CHEMOTHERAPEUTIC DRUGS ON NORMAL JAW BONES USING OSTEOPONTIN: AN EXPERIMENTAL STUDY.
}

\author{
Amina Fouad Farag ${ }^{1}$, Nadia Fathy Hassabou ${ }^{2}$, Yasmine Alaa El-Din ${ }^{3}$ and Ahmed Nabil Fahmi ${ }^{4}$ \\ 1. Assistant Lecturer of Oral Pathology, Faculty of Dentistry, October 6 University, Egypt. \\ 2. Lecturer of Oral Pathology, Faculty of Dentistry, October 6 University, Egypt. \\ 3. Assistant Lecturer of Oral Pathology, Faculty of Dentistry, October 6 University, Egypt. \\ 4. Lecturer of Oral Pathology, Faculty of Dentistry, BaniSuef University, Egypt.
}

\section{Manuscript Info}

Manuscript History

Received: 06 November 2017

Final Accepted: 08 December 2017

Published: January 2018

Key words:-

5-fluorouracil, methotrexate, osteoblasts, transcription factors, osteopontin.

\begin{abstract}
Background: Chemotherapy does not differentiate between rapidly dividing cancer cells and normal cells that divide at higher rates as bone forming cells. Methotrexate and 5-fluorouracil are chemotherapeutic drugs with high potentiality to damage bone forming cells by blocking their DNA synthesis which leads to their suppression and in turn impaired bone formation mainly by dysregulation of Runx 2 and osterix associated with osteoblastic cell differentiation. Aim: Compare the toxic effects of methotrexate and 5-flurouracil on normal bone cells. Material and methods: A total of 30 rats were divided into three groups. Group I was control group, Group II was given a single $20 \mathrm{mg} / \mathrm{kg}$ intraperitoneal dose of MTX and Group III was given a single $150 \mathrm{mg} / \mathrm{kg}$ intraperitoneal dose of $5-\mathrm{FU}$. Four days later, the rats were euthanized and their mandibles were dissected, immediately fixed in $10 \%$ formalin, processed and prepared for histopathological and immunohistochemical examinations using osteopontin. Statistical analysis was performed using ANOVA test where $\mathrm{P}$ values $<0.05$ were statistically significant. Results: Variable degenerative effects on the bone forming cells were observed where such effects were higher in 5FU group compared to the MTX group. Positive osteopontin expression and higher number of bone formative cells were detected in the control group followed by MTX group then the 5-FU group. Low osteopontin expression was correlated with decreased number of bone cells and subsequent decreased jaw bone density and formation. Conclusion: 5FU is more cytotoxic to the normal bone forming cells than MTX.
\end{abstract}

Copy Right, IJAR, 2018,. All rights reserved.

\section{Introduction:-}

Chemotherapy is one of the most common modalities in cancer treatment. Although the toxicity of chemotherapeutic drugs is directed against cancer cells, they show several side effects on the normal healthy cells especially the actively dividing ones leading to their damage (Saini et al., 2012).

The most successful chemotherapeutic regimens are associated with high intensive dose where there is direct proportion between the toxicity whether acute and chronic and the doses used to treat many human cancers 
(Curigliano et al., 2012). Therefore, it is important to determine the most effective dose at which tumor will respond to therapy with minimal adverse effects on normal tissues (Hensley et al., 2009).

Tissues with higher rates of cellular proliferation such as neoplasms, epidermis, bone marrow and the lining of the oral cavity are considered as the most sensitive to the effects of chemotherapeutic drugs (Deeming et al., 2005 and Yüncü et al., 2006).

Methotrexate (MTX) and 5-fluorouracil (5-FU) are common chemotherapeutic drugs widely used in the treatments of many malignancies. They are classified as antimetabolites interfering with the formation of biomolecules within the cell including the nucleotides which are the building blocks of DNA. Therefore, they hinder DNA replication, gene transcription and cell division. These drugs cause cell cycle arrest in tissues by blocking DNA synthesis which in turn leads to hypoproliferation followed by impaired cellular integrity and apoptosis (Marks \& Edwards, 2012 and Thomas et al., 2016).

Osteoblasts and preosteoblasts are directly affected by the cytotoxicity of chemotherapeutic drugs resulting in suppressed cell proliferation and cell death. Transcription factors are an important target for cancer therapy through newly developed delivery systems that specifically target tumor cells (Yeh et al., 2013 and Xiana et al., 2004).

Runx2 and osterix (Osx) are the main transcription factors that stimulate osteoblastic cell differentiation that dysregulated by toxic effect of chemotherapeutic drugs. They bind to promoters of osteoblast specific genes such as Col1 $\alpha 1$, BSP and osteopontin which upregulate gene transcription (Sinha \& Zhou, 2013).

Extracellular matrix proteins such as osteopontin (OPN) expressed in bone predominantly by osteoblasts and osteocyctes (bone forming cells) as well as osteoclasts (bone resorbing cells). OPN could play a role in mediation of signals to osteoblastic differentiation and its expression was enhanced during the bone remodeling as it considered one of the positive factors for the bone formation (Huang et al., 2013).

Our approach was to compare the negative effect of both chemotherapeutic drugs on bone cells. Therefore, we examined the expression of OPN in experimental rats after administration of drugs to evaluate their effects on osteoblastic activity in jaw bones.

\section{Materials and Methods:-}

A total of 30 adult male albino rats (weighing 200-250 grams) were used. The animals were divided randomly into three groups of 10 rats each. They were housed in cages under constant conditions of temperature, ventilation and were maintained on a basic diet of regular rat chow, distilled water and libitum.

Group I (Control group):- received $1 \mathrm{ml}$ sterile normal saline,

Group II (MTX group):- received a single intraperitoneal injection of $150 \mathrm{mg} / \mathrm{kg}$ of MTX and

Group III (5-FU group): received a single intraperitoneal injection of $150 \mathrm{mg} / \mathrm{kg}$ of 5-FU.

Both drugs were supplied in a solution form by EBEWE PHARMA, Unterach, Austria where each vial of MTX contained $1000 \mathrm{mg} / 10 \mathrm{ml}$ while each ampoule of 5 -FU contained $500 \mathrm{mg} / 10 \mathrm{ml}$.

The animals of both the experimental and control groups were euthanized on day 4 by tripling the anesthetic dose of ketamine base as lethal dose (Close et al., 1997). The protocol was followed and approved by the Animal Ethics Committee of Faculty of Science Cairo, University. Then they were dissected and the mandibules were removed and fixed in $10 \%$ buffered formalin.

After fixation, they were embedded in paraffin and three tissue sections of $4 \mu \mathrm{m}$ thickness were cut from both control and experimental groups where one tissue section mounted on ordinary glass slide and stained with hematoxylin and eosin for histopathological examination.

The other two were mounted on positively charged glass slides for immunohistochemical analysis with OPN (mouse, monoclonal clone, AKm2A1, Santa Cruz Biotechnology). 
Image Analysis for area percentage with positive reaction to OPN was performed using the Leica Q550 IW Imaging Workstation. This was done along the periosteium in five microscopic fields with the most uniformly positive immunohistochemical reaction at a magnification of x200 (figs. 3a, b \&c ).

Statistical analysis was done with SPSS (Statistical Package for Social Sciences) 13.0 software. All the obtained data were given as mean $\pm \mathrm{SD}$. One-way variance test (ANOVA) was used to compare between the groups. P values $<0.05$ were accepted as statistically significant.

\section{Results:-}

Histopathological Findings:-

Microscopic examination of the jaw bone of control group revealed Haversian systems with highly cellular lacunae containing osteocytes interconnecting with each other and to those in circumferential layers of bone that underlie the highly cellular periosteum through their cytoplasmic processes that extend into the bony canaliculi (fig. 1a).

Four days after the MTX injection, there was distortion of the lamellar architecture and degeneration of a number of the bone formative cells lining the periosteal surface together with several empty lacunae due to osteocyte degeneration (fig. 1b).

As with MTX, 5-FU revealed similar toxic degenerative effects on the formative bone cells four days after its injection but such degenerative effects were more evident and much higher in severity in 5-FU group compared to the MTX group (fig. 1c).

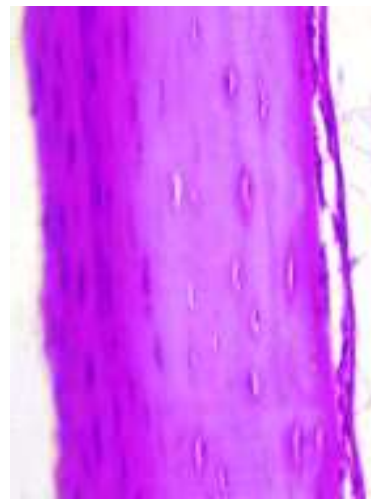

(1a)

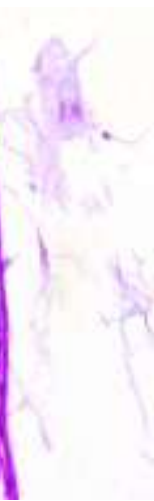

(1b)

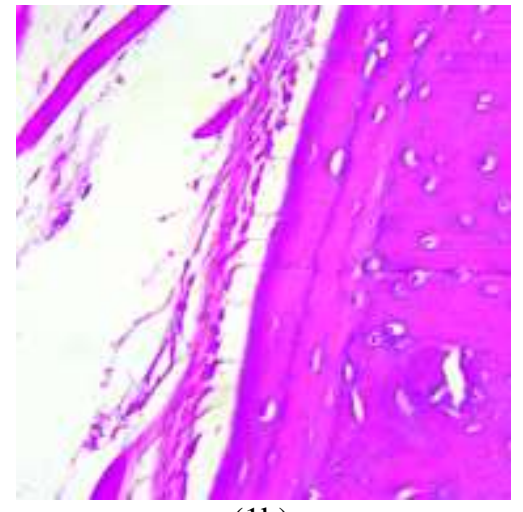

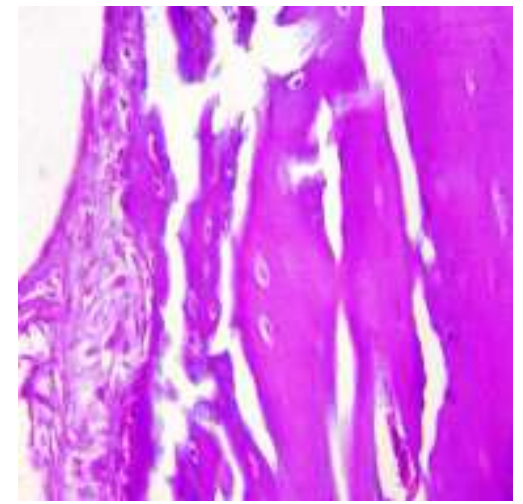

(1c)

Fig. 1:- Jaw bones of rats stained with routine H\&E stain at a magnification of x200 (a) Control group (b) MTX group after 4 days of injection and (c) 5-FU group after 4 days of injection, (H\&E, x200)

\section{Immunohistochemical Findings:-}

Immunohistochemical examination of the control group revealed strong positive OPN immunoexpression compared to both experimental groups (fig. 2a).

MTX group showed higher and stronger expression than the 5-FU group reflecting more degenerative changes were associated with 5-FU compared to MTX administration (figs. $2 \mathrm{~b} \& \mathrm{c}$ ).

Measurement of area\% of similar intensity OP-immunostained bone formative cells revealed higher bone formative cells per $\mu \mathrm{m}^{2}$ of bone surface area (area \%) were given by the control group followed by MTX group and the 5-FU showed the lower values (figs. 3a, b \& c, table 1). 


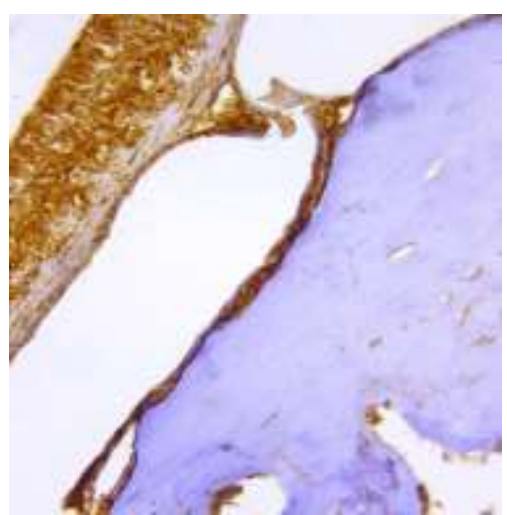

(2a)

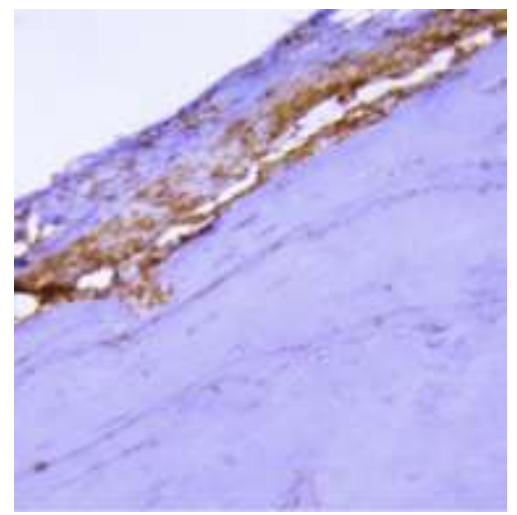

(2b)

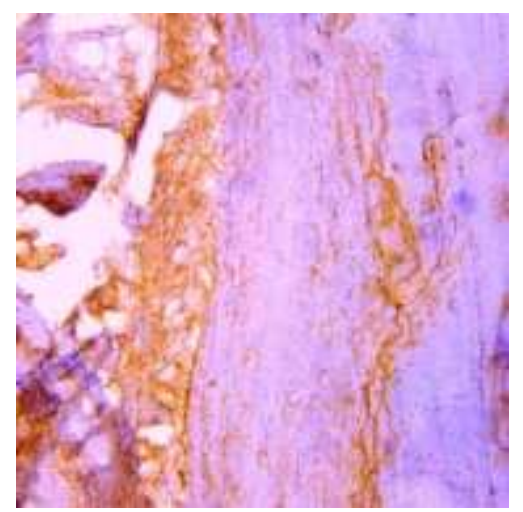

(2c)

Fig. 2:-Jaw bones of rats stained with OP immunostain at a magnification of x200 (a) Control group showing strong positive OP staining (b) MTX group after 4 days of injection showing moderate positive OP staining and (c) 5-FU group after 4 days of injection showing weak positive OP staining (OP antibody, x200).

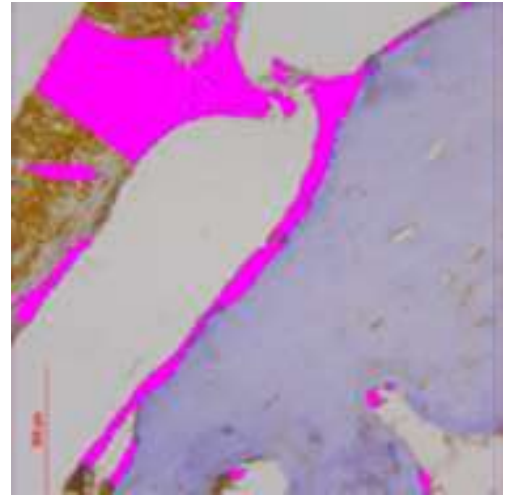

(3a)

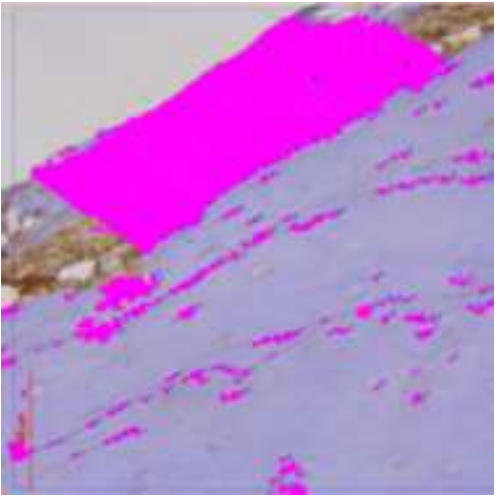

(3b)

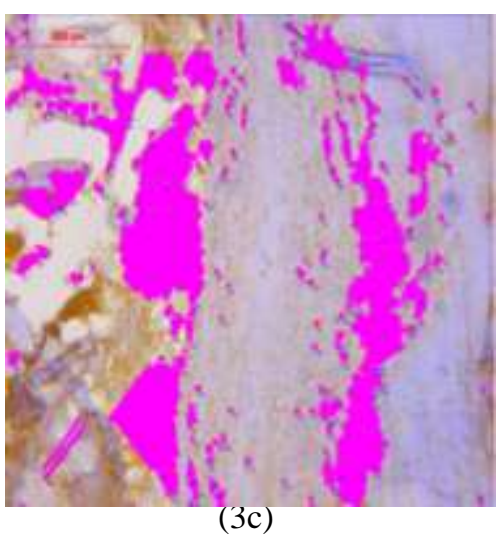

Fig. 3:- Measurement of area\% of similar intensity OP-immunostained bone formative cells using image analyzer computer system in (a) control, (b) MTX and (c) 5-FU respectively.

\section{Statistical Analyses:-}

Significant difference in mean values of OPN expression that represent the area $\%$ of bone formative cells (number of cells per $\mu \mathrm{m}^{2}$ bone surface area perimeter) was seen between the control group, MTX group and 5-FU group (table 1, graph 1).

Table 1:- Difference in mean OPN area \% of bone formative cells between control group, MTX group and 5-FU group using ANOVA test

\begin{tabular}{|c|c|c|c|}
\hline \multirow{2}{*}{ Group } & \multicolumn{3}{|c|}{ Area \% } \\
\hline & $\mathbf{M} \pm \mathbf{S d}$ & f-Value & p-Value \\
\hline Control & $5147.97 \pm 11809.99$ & \multirow{3}{*}{3.25776} & \multirow{3}{*}{$0.040373 *$} \\
\hline MTX & $2922.67 \pm 9067.95$ & & \\
\hline 5-FU & $1500.12 \pm 2354.99$ & & \\
\hline
\end{tabular}

$*$ = significant, $(\mathrm{p}<0.005)$ 


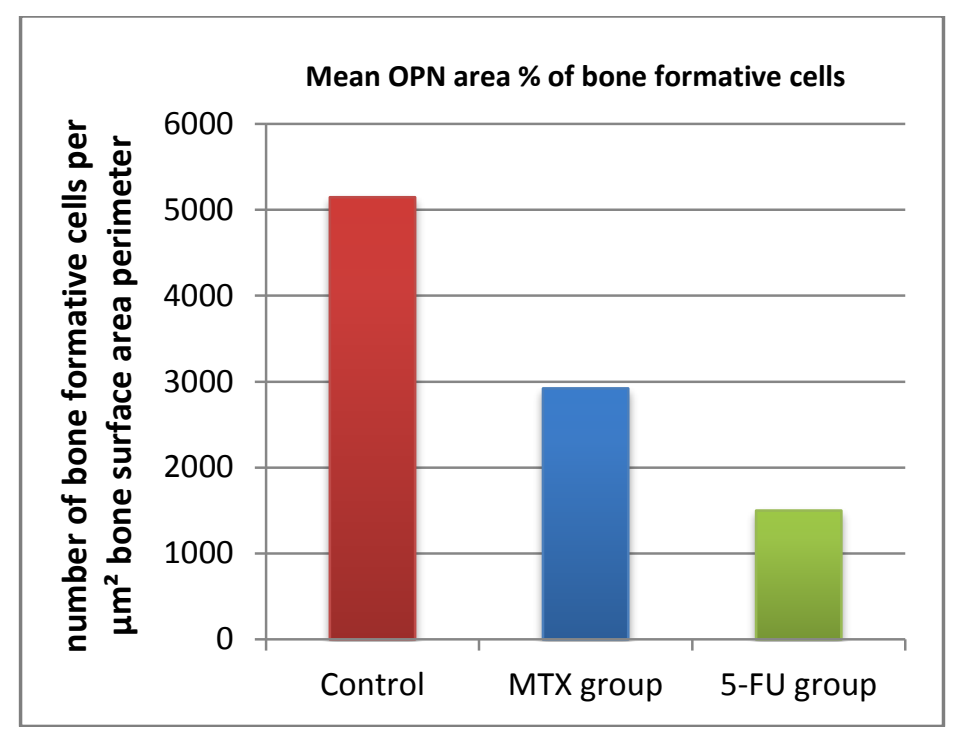

Graph 1:- Mean OPN area \% of number of bone formative cells bone formative cells per $\mu \mathrm{m}^{2}$ bone surface area between control, MTX and 5-FU groups.

\section{Discussion:-}

Chemotherapy considered as a great treatment modality using chemical agents to stop the growth and eliminate cancer cells even at distant sites from the origin of primary tumor. However, the main obstacle is that it does not distinguish between cancer cells and normal cells and eliminates not only the fast-growing cancer cells but also other fast-growing cells in the body (El-Sayyad et al., 2009).

MTX and 5-FU are considered as antimetabolites used as chemotherapeutic drugs. Although, they generate acceptable outcome in chemotherapy of some cancers, they also exhibit severe toxicity and undesirable side effects. Furthermore, the effects of these drugs on bone growth and formation remain unknown (Smane \&Pilmane, 2016). That is the main aim of this study to assess the potential effect on normal jaw bone of rats by detection of immunohistochemical expression of OPN among control and experimental groups of rats received both drugs.

Regarding OPN, it is considered as a prominent bone matrix protein that is produced by differentiated osteoblasts, osteocytes and also by osteoclasts. It is also involved in many physiological and pathological processes including cell adhesion, angiogenesis, apoptosis, inflammatory responses and tumor metastasis. Consistent with a multiplicity of functions for OPN, it is involved specifically in both the forming and resorption of bone. That is why its suppression causes defects in process of bone remodeling (Fan et al., 2016)

In the current work, it was observed that lower values of OPN positive immunoreactivity were in 5-FU group reflecting the marked decrease in the activity and/or number of osteoblasts. This finding was in accordance with the results of Xiana et al. (2004) and Xian et al. (2006) who proofed that 5-FU caused reduction in both osteoblast and preosteoblast density.

This is further supported by the findings of Theodoro et al. (2017) who claimed that 5-FU chemotherapy affects bone growth directly by inducing apoptosis and inhibiting proliferation of bone cells through dysregulation of transcriptional factors and blocking DNA synthesis. Therefore, 5-FU chemotherapy directly impairs the mechanism of bone growth and osteoblastic differentiation.

Concerning MTX, the current results demonstrated lower values of OPN than the control group but it was higher than 5-FU group. These findings were in agreement with Xian et al. (2007) and Pountos et al., (2017) who agreed that effect of MTX chemotherapy on bone metabolism cause inhibitory effect on osteoblastic function without altering its numbers.

It was believed that rat models of MTX chemotherapy also demonstrated a reduction in bone marrow osteoprogenitor cells, suppressed stromal progenitor cell proliferation and decreased osteoblastic function in dose dependent manner (Fan et al., 2009). 
The result of present work came in line with the study of Xian et al. (2008) who found that MTX had no obvious effects on apoptosis but only some moderate damaging effects on proliferation of osteoblasts and preosteoblasts in bone with moderate changes in osteoblastic cell density along trabecular surface in comparing to 5-FU.

\section{Conclusion and Future Recommendations:-}

In conclusion, 5-FU revealed low OPN expression which correlated with decreased number of formative (osteoblasts) cells and subsequent decreased jaw bone density and formation compared to MTX.

We recommend further research on chemotherapeutic agents to identify the drugs with the least side effects on normal tissues and more specific to cancer cells. Cautions should be considered in using MTX and 5-FU especially in young aged children due to its great destructive side effects on the process of bone remodeling especially bone formation.

\section{Acknowledgement:-}

We would like to thank all the members of the Research Centers in Faculty of Dentistry, Cairo University and in the Faculty of Dentistry, MSA University for the facilities they provided to the authors during the course of this work.

\section{Conflict of Interest:-}

The authors declare that they have no conflict of interest.

\section{Referrences:-}

1. Basilio de Oliveira, R. and Pannain, V. (2015): Prognostic angiogenic markers (Endoglin, VEGF, CD31) and tumor cell proliferation (Ki67) for gastrointestinal stromal tumors. World J. Gastroenterol., 21(22):6924-6930.

2. Saini, R., Chouhan, R., Bagri, L. and Bajpai,A. (2012): Strategies of Targeting Tumors and Cancers. J. Can. Res. Updates, 1:129-152.

3. Curiglian, G., Cardinale, D., Suter, T., Plataniotis, G., de Azambuja, E., Sandri, M., et al. (2012): Cardiovascular toxicity induced by chemotherapy, targeted agents and radiotherapy: ESMO Clinical Practice Guidelines. Ann. Oncol., 23(7):vii155-166.

4. Hensley, M., Hagerty, K., Kewalramani, T., Green, D., Meropol, N., Wasserman, T. et al. (2009): American Society of Clinical Oncology 2008 clinical practice guideline update: Use of chemotherapy and radiation therapy protectants. J. Clin. Oncol., 27(1):127-145.

5. Yüncü, M., Eralp, A. and Celik, A. (2006): Effect of aged garlic extract against methotrexate-induced damage to the small intestine in Jun rats. Phytother. Res., 20(6):504-10, 2006.

6. Deeming, G., Collingwood, J. and Pemberton, M. (2005): Methotrexate and oral ulceration. Br. Dent. J., 198(2):83-85.

7. Marks, J. and Edwards, C. (2012): Protective effect of methotrexate in patients with rheumatoid arthritis and cardiovascular comorbidity. Ther. Adv. Musculoskelet. Dis., 4(3):149-157.

8. Thomas, S., Grami, Z., Mehta, S. and Patel, K. (2016): Adverse effects of 5-fluorouracil: Focus on rare side effects. Cancer Cell Microenviron., 3(2):e1266.

9. Yeh, J., TONIOLO, P. AND FRANK, D. (2013): TARGETING TRANSCRIPTION FACTORS: PROMISING NEW STRATEGIES FOR CANCER THERAPY. CURR. OPIN. ONCOL., 25(6):652-658.

10. Xiana, C., Howarth, G., Cool J. and Fostera, B. (2004): Effects of acute 5-fluorouracil chemotherapy and insulin-like growth factor- I pretreatment on growth plate cartilage and metaphyseal bone in rats. Bone, 35(3):739-749.

11. Sinha, K. and Zhou, X. (2013): Genetic and molecular control of Osterix in skeletal formation. J. Cell Biochem., 114 (5):975-984.

12. Huang, W., Yang, S., Shao, J. and Li, Y. (2013): Signaling and transcriptional regulation in osteoblast commitment and differentiation. Front. Biosci., 12:3068-3092.

13. Close, B., Banister, K., Baumans, V., Bernoth, E., Bromage, N., Bunyan, J., et al. (1997): Recommendations for euthanasia of experimental animals: Part 2 Working party. Lab. Anim., 31(1):1-32.

14. El-Sayyad, H., Ismail, M., Shalaby, F., Abou-El-Magd, R., Gaur, R., Fernando, A., et al. (2009): Histopathological effects of cisplatin, doxorubicin and 5-flurouracil (5-FU) on the liver of male albino rats. Int. J. Biol. Sci., 5(5): 466-473.

15. Smane, L. and Pilmane, M. (2016): Osteopontin, osteocalcin and osteoprotegerin expression in human tissue affected by cleft lip and palate. Int. Conf. Society Health Welfare 2014, SHS Web of Conferences, 30:00008. 
16. Fan, C., Georgiou, K., McKinnon, R., Keefe, D., Howe, P. and Xian, C. (2016): Combination chemotherapy with cyclophosphamide, epirubicin and 5-fluorouracil causes trabecular bone loss, bone marrow cell depletion and marrow adiposity in female rats. J. Bone. Miner. Metab., 34(3):277-291.

17. Xian, C., Cool, J., Pyragius, T. and Foster, B. (2006): Damage and recovery of the bone growth mechanism in young rats following 5-fluorouracil acute chemotherap.y. J Cell Biochem., 99(6):1688-1704.

18. Xian, C., Cool, J., Scherer, M., Macsai, C., Fan, C., Covino, M. et al. (2007): Cellular mechanisms for methotrexate chemotherapy-induced bone growth defects. Bone, 41(5):842-850.

19. Xian, C., Cool, J., Scherer, M., Fan, C. and Foster, B. (2008): Folinic acid attenuates methotrexate chemotherapy-induced damages on bone growth mechanisms and pools of bone marrow stromal cells. J. Cell Physiol., 214(3):777-785.

20. Fan C., Cool, J., Scherer, M., Foster, B., Shandala, T., Tapp, H., et al. (2009): Damaging effects of chronic low dose methotrexate usage on primary bone formation in young rats and potential protective effects of folinic acid supplementary treatment. Bone, 44(1):61-70.

21. Pountos, I. and Giannoudis, P. (2017). Effect of methotrexate on bone and wound healing. Expert Opin. Drug Saf., 16(5):535-545. 